\title{
An Optimal Power Allocation Scheme for the STC Hybrid-ARQ over Energy Limited Networks
}

\author{
Hongbo Liu, Member, IEEE, Leonid Razoumov, Member, IEEE, Narayan Mandayam, Fellow, IEEE, \\ and Predrag Spasojević, Member, IEEE
}

\begin{abstract}
In this paper, we show that for STC (Space-Time Coded) Hybrid-ARQ (Automatic Repeat reQuest) schemes with (re)transmission power control over independent Rayleigh block fading channels, the problem of optimizing energy efficiency with a PER (Packet Error Rate) constraint can be solved as a geometric programming problem. The optimum transmit power increases super-linearly with each requested retransmission and the fraction of the average power optimally allocated to each ARQ round only depends on the incremental diversity gain. The energy savings increases with a decrease in the PER targets and decreases with an increase in the diversity gain.
\end{abstract}

Index Terms-Energy limited networks, geometric programming, hybrid ARQ, space-time codes.

\section{INTRODUCTION}

$\mathbf{F}$ OR energy limited data networks, e.g., sensor networks, one of the goals is to deliver as much information as possible with a limited energy supply and a maximum delay constraint. For data transmissions with loose delay constraints, the ARQ (Automatic Repeat reQuest) scheme is often used in addition to an FEC (Forward Error Correction) scheme, which is known as Hybrid-ARQ, to provide adaptive data rates, and hence improve the energy efficiency. In the following, we refer to the uncoded information message as a "source packet" and the successive transmissions of the coded blocks of the same source packet as "ARQ rounds".

The traditional Hybrid-ARQ scheme is a constant power transmission scheme. A few power adaptation schemes have been proposed to increase the throughput [1], reduce energy consumption [2], or provide extra diversity gain [3] by making the transmit power of the $l$-th ARQ round a function of $l$. In [3], for long-term static MIMO (Multiple Input Multiple Output) channels, i.e., the channel coefficients remain constant during all ARQ rounds and change to new independent values with each new source packet, an asymptotically optimal power control algorithm is proposed that yields very significant diversity advantage. However, for short-term static channels, where the channel coefficients remain constant during each

Manuscript received March 8, 2008; revised October 24, 2008 and March 26, 2009; accepted June 2, 2009. The associate editor coordinating the review of this letter and approving it for publication was M. Torlak.

H. Liu, N. Mandayam, and P. Spasojević are with WINLAB, Department of Electrical and Computer Engineering, Rutgers University, North Brunswick, NJ 08902 USA (e-mail: \{hongbol, narayan, spasojev\}@ @inlab.rutgers.edu).

L. Razoumov is with AT\&T Labs, 180 Park Ave, Florham Park, NJ 07932, USA (e-mail: leor@research.att.com).

The material in this paper was presented in part at Conference on Information Sciences and Systems (CISS) 2004 and CISS 2005. This work was supported in part by the NSF under Grant No. FMF 0429724.

Digital Object Identifier 10.1109/TWC.2009.12.080335
ARQ round and change independently for the next ARQ round, no optimal power adaptation scheme is proposed.

Different from previous works, we propose a power adaptation algorithm where the power level at each ARQ round is optimized for the short-term static channel variation based on the long term channel statistics. Our goal is to choose the transmit power for the $l$-th ARQ round to minimize the average energy consumption under a PER (Packet Error Rate) constraint with the maximal number of ARQ rounds to be fixed to $L$ over an $L$-block Rayleigh fading STC (Space-Time Coded) channel. We show that our problem can be formulated and solved as a GP (Geometric Programming) problem and a closed-form solution can be obtained. We also show that the optimized transmit power increases in a super-linear manner each time a retransmission is requested and the fraction of the average energy optimally allocated to the $l$-th ARQ round only depends on the incremental diversity gain.

This paper is organized as follows: In Section II, we provide the channel model and some preliminaries used in this work. In Section III, we show how the optimization problem can be formulated and solved as a GP problem. In Section IV, simulation validations for the optimal power allocation strategy are presented and we summarize our work in Section V.

\section{Channel Model And the Preliminaries}

\section{A. Channel Model for Hybrid-ARQ}

There are several variations of the Hybrid-ARQ schemes, i.e., type I [4], type II with IR (Incremental Redundancy) [5] and Chase combining [6]. In this paper, an error-free and zerodelay ARQ feedback channel is assumed for all ARQ schemes. If a decoding error occurs, a one-bit retransmission request (NACK) is sent back to the transmitter.

We consider a frequency flat fading channel with no CSI (channel state information) at the transmitter and perfect CSI at the receiver. For a STC channel with $M_{T}$ transmit antennas and $M_{R}$ receive antennas, the channel state matrix $\mathbf{H}_{l} \in$ $\mathbb{C}^{M_{R} \times M_{T}}, l=1, \cdots, L$ remains constant during the $l$-th ARQ round and changes independently for different ARQ rounds. Each ARQ round takes $T$ channel uses. Define $\mathbf{x}_{l, t} \in \mathbb{C}^{M_{T}}$, $\mathbf{y}_{l, t} \in \mathbb{C}^{M_{R}}$, and $\mathbf{w}_{l, t} \in \mathbb{C}^{M_{R}}, l=1, \cdots, L, t=1, \cdots, T$ as the transmitted signal, received signal, and corresponding channel noise vector, respectively. The channel noise is assumed to be temporally and spatially white with i.i.d. complex Gaussian entries $\sim \mathcal{C N}\left(0, N_{0}\right)$. Let $E_{l}$ be the transmit power per channel use, which remains constant for the $l$-th ARQ 
round, and the energy consumption for the $l$-th ARQ round is $E_{l} T$. The channel model is defined as

$$
\mathbf{y}_{l, t}=\sqrt{\frac{E_{l}}{M_{T}}} \mathbf{H}_{l} \mathbf{x}_{l, t}+\mathbf{w}_{l, t},
$$

which corresponds to a short term static channel in [3]. It is also referred to as the $L$-block fading Hybrid-ARQ model in this paper.

A power allocation scheme for an $L$-block fading HybridARQ is defined as

$$
\mathbf{E}=\left(E_{1}, E_{2} \cdots, E_{L}\right)=\left(\rho u_{1}, \rho u_{2}, \cdots, \rho u_{L}\right)=\rho \mathbf{u},
$$

where $\rho=\|\mathbf{E}\|$ and $\mathbf{u}=\frac{\mathbf{E}}{\|\mathbf{E}\|}$, and $\|\cdot\|$ represents the norm of the vector.

\section{B. Average Power}

In this paper, the average power is defined as the average energy normalized by $T$. Define the probability of the event that a NACK is received at the $l$-th round as $P_{l}$, and let $P_{0}=1$ by definition. Let $\mathbb{E}[\cdot]$ denote the expectation of a random variable. Following [3], the average power per source packet is

$$
\overline{\mathbf{E}}=\mathbb{E}\left[\sum_{l=1}^{\mathcal{T}} \mathbf{E}_{l}\right]=\sum_{l=1}^{L} \mathbf{E}_{l} P_{l-1},
$$

where $\mathcal{T}$ is a random number of retransmissions for each source packet.

\section{Diversity Gain}

The optimal diversity-multiplexing tradeoff for ARQ MIMO channels has been proposed in [3]. In this paper, we use a different approach to obtain the diversity gain. For an $L$ block fading Hybrid-ARQ, we define the term incremental diversity gain. For a given power allocation scheme $\mathbf{E}=\rho \mathbf{u}$, the diversity gain at the $l$-th ARQ round is defined as

$$
d_{l}=-\lim _{\rho \rightarrow \infty} \frac{\log P_{l}(\rho \mathbf{u})}{\log \rho},
$$

where $P_{l}(\rho \mathbf{u})$ is the accumulative PER after the $l$-th ARQ round. The incremental diversity gain, which represents the diversity gain corresponding to the $l$-th ARQ round, is defined here as

$$
D_{l}=d_{l}-d_{l-1}=-\lim _{\rho \rightarrow \infty} \frac{\log P_{l}(\rho \mathbf{u})-\log P_{l-1}(\rho \mathbf{u})}{\log \rho},
$$

where $d_{0}=0$ by definition.

\section{Probability of Errors in Hybrid-ARQ}

The average energy consumption is closely related to the PER. For each ARQ round, the CSI is unknown to the sender, and therefore, the results for the open-loop PER analysis can be applied. We can derive the PER for both type I and type II $L$-block fading Hybrid-ARQ model based on the analysis for STC in [7].

Let $\mathcal{E}_{l}$ denote the event that a decoding error occurs at the $l$ th ARQ round. We assume that the undetected decoding error can be ignored. Given a sequence of channel state matrices

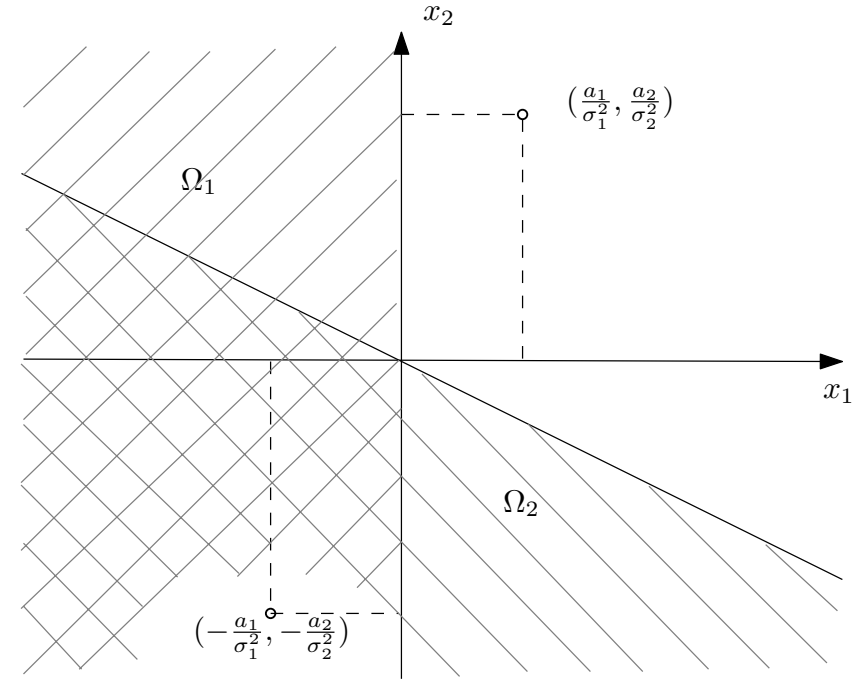

Fig. 1. Error event decision regions.

$\mathbf{H}_{1}, \mathbf{H}_{2}, \cdots, \mathbf{H}_{L}$, the probability of sending a NACK at the $l$-th ARQ round for $l=1, \cdots, L$ is given in equation (4).

$$
\begin{array}{r}
P_{l}\left(\rho \mathbf{u} \mid \mathbf{H}_{1}, \mathbf{H}_{2}, \cdots \mathbf{H}_{l}\right)=\operatorname{Pr}\left(\mathcal{E}_{1} \mid \mathbf{H}_{1}\right) \operatorname{Pr}\left(\mathcal{E}_{2} \mid \mathcal{E}_{1}, \mathbf{H}_{1}, \mathbf{H}_{2}\right) \cdots \\
\operatorname{Pr}\left(\mathcal{E}_{l} \mid \mathcal{E}_{1}, \mathcal{E}_{2}, \cdots, \mathcal{E}_{l-1}, \mathbf{H}_{1}, \mathbf{H}_{2}, \cdots \mathbf{H}_{l}\right) \\
=\operatorname{Pr}\left(\mathcal{E}_{1}, \cdots, \mathcal{E}_{l} \mid \mathbf{H}_{1}, \mathbf{H}_{2}, \cdots \mathbf{H}_{l}\right) .
\end{array}
$$

We use superscripts $I$ and $I I$ to denote the type $I$ and the type II Hybrid-ARQ respectively. For type I Hybrid-ARQ, only the latest received packet is used for decoding. For Chase combining and type II Hybrid-ARQ, the code blocks from the ARQ round 1 through $l$ are all combined and decoded as one codeword using a maximum likelihood (ML) decoder. The Chase combining Hybrid-ARQ is considered a special case of the type II Hybrid-ARQ and, therefore, is not discussed separately.

Proposition 1: The error probability of an $L$-block fading type II Hybrid-ARQ after the $l$-th ARQ round has the same diversity order as the error probability of decoding a codeword with $l$ fading blocks at the $l$-th ARQ round alone without considering decoding errors at previous ARQ rounds, i.e.,

$$
d_{l}=-\lim _{\rho \rightarrow \infty} \frac{\log \operatorname{Pr}\left(\mathcal{E}_{1}, \cdots, \mathcal{E}_{l}\right)}{\log \rho}=-\lim _{\rho \rightarrow \infty} \frac{\log \operatorname{Pr}\left(\mathcal{E}_{l}\right)}{\log \rho} .
$$

Proof: For an $L$-block fading type II Hybrid-ARQ with maximum likelihood (ML) decoding rule, the error probability can be bounded as

$$
\begin{aligned}
\frac{1}{2^{l-1}} \operatorname{Pr}\left(\mathcal{E}_{l} \mid \mathbf{H}_{1}, \mathbf{H}_{2}, \cdots \mathbf{H}_{l}\right) & \leq \operatorname{Pr}\left(\mathcal{E}_{1}, \cdots, \mathcal{E}_{l} \mid \mathbf{H}_{1}, \mathbf{H}_{2}, \cdots \mathbf{H}_{l}\right) \\
& \leq \operatorname{Pr}\left(\mathcal{E}_{l} \mid \mathbf{H}_{1}, \mathbf{H}_{2}, \cdots \mathbf{H}_{l}\right) .
\end{aligned}
$$

The upper bound is obvious because the probability of the joint event is equal to or less than the probability of an individual event. The lower bound can be obtained based on the properties of the Euclidean distance based ML decoding as shown in Fig. 1, where $x_{i}=\left|\mathbf{x}_{i}\right|, a_{i}=\left|\mathbf{s}_{i}\right|, i=1, \cdots, l$, and $\mathbf{x}_{i}$ is the received signal, $\mathbf{s}_{i}$ is a super symbol composed of all different symbols between two codewords, $\Omega_{l}$ is the error decision region, and $\sigma_{i}$ is the noise power at the $i$-th ARQ 
round. We have

$$
\begin{aligned}
& \operatorname{Pr}\left(\mathcal{E}_{1}, \mathcal{E}_{2} \mid \mathbf{H}_{1}, \mathbf{H}_{2}\right)=\int_{\Omega_{1} \cap \Omega_{2}} p\left(x_{1}, x_{2}\right) d x_{1} d x_{2} \\
& \geq \frac{1}{2} \int_{\Omega_{2}} p\left(x_{1}, x_{2}\right) d x_{1} d x_{2} \\
& =\frac{1}{2} \int_{\Omega_{2}} p\left(y_{2}\right) d y_{2}=\frac{1}{2} \operatorname{Pr}\left(\mathcal{E}_{2} \mid \mathbf{H}_{1}, \mathbf{H}_{2}\right),
\end{aligned}
$$

with change of variables $y_{2}=\sqrt{\frac{x_{1}^{2}}{\sigma_{1}^{2}}+\frac{x_{2}^{2}}{\sigma_{2}^{2}}}$. Equation (7) is based on the property of multi-dimensional Gaussian distribution. With the same analysis, it follows that

$$
\begin{aligned}
& \operatorname{Pr}\left(\mathcal{E}_{1}, \mathcal{E}_{2}, \cdots, \mathcal{E}_{l} \mid \mathbf{H}_{1}, \mathbf{H}_{2}, \cdots \mathbf{H}_{l}\right)=\int_{\bigcap_{k=1}^{l} \Omega_{l}} p(\mathbf{x}) d \mathbf{x} \\
& \geq \frac{1}{2^{l-1}} \int_{\Omega_{l}} p(\mathbf{x}) d \mathbf{x}=\frac{1}{2^{l-1}} \operatorname{Pr}\left(\mathcal{E}_{l} \mid \mathbf{H}_{1}, \mathbf{H}_{2}, \cdots \mathbf{H}_{l}\right) .
\end{aligned}
$$

Taking the expectation over all channel states for equation (6), equation (5) then follows.

Proposition 2: The maximum diversity order of an $L$ block fading Hybrid-ARQ channel is equal to $M_{T} M_{R} L$ and the maximum incremental diversity is $D_{1}=D_{2}=\cdots=$ $D_{L}=M_{T} M_{R}$.

Proof: For the type I Hybrid ARQ, all error events $\mathcal{E}_{l}$ are mutually independent, therefore, the PER at the $l$-th ARQ round is

$$
\begin{aligned}
& P_{l}^{I}(\mathbf{E})=\mathbb{E}\left[P_{l}^{I}\left(\mathbf{E} \mid \mathbf{H}_{1}, \mathbf{H}_{2}, \cdots \mathbf{H}_{l}\right)\right] \\
& =\prod_{k=1}^{l} \mathbb{E}\left[\operatorname{Pr}\left(\mathcal{E}_{k} \mid \mathbf{H}_{k}\right)\right]=\prod_{k=1}^{l} \operatorname{Pr}\left(\mathcal{E}_{k}\right)=\prod_{k=1}^{l} \operatorname{Pr}\left(E_{k}\right)
\end{aligned}
$$

where $\operatorname{Pr}\left(E_{k}\right)$ is the PER for the $k$-th ARQ round. By applying [7, equation (13)], the asymptotic PER bound of the type I Hybrid-ARQ after the $l$-th ARQ round is

$$
P_{l}^{I}(\mathbf{E}) \leq \prod_{k=1}^{l}\left\{\frac{1}{4}\left[\frac{E_{k}}{4 N_{0}}\right]^{-\eta_{\min } \cdot M_{R}} \sum_{\mathbf{e} \in \mathcal{E}_{\min }}\left[\prod_{i=1}^{\eta_{\min }} \lambda_{i}\right]_{(10)}^{-M_{R}}\right\},
$$

where $\mathbf{e}$ is the error sequence, $\mathcal{E}_{\min }$ is the set of error sequences with minimum diversity, $\lambda_{i}$ is the eigenvalues derived from the STC channel matrix [7, equation (13)], and $\eta_{\min }$ is the minimum diversity of a single block fading channel. With optimum code design, maximum $\eta_{\min }=M_{T}$ is achieved, and Proposition 2 follows.

For the type II Hybrid-ARQ, based on Proposition 1, to obtain the maximum diversity order and the incremental diversity gain, we only need to consider the decoding error corresponding to an $l$ block fading channel. To obtain the PER with a power allocation scheme $\mathbf{E}=\left(\rho u_{1}, \rho u_{2}, \cdots, \rho u_{L}\right)$ over an $l$ block fading channel, we consider an equivalent transmission scheme, in which the transmit power is $\rho$ for each transmission while the fading gain is scaled by $u_{k}$ for the $k$-th fading block. The asymptotic PER bound of the type II Hybrid-ARQ after the $l$-th ARQ round is [7, equation (13)]

$P_{l}^{I I}(\mathbf{E}) \leq \frac{1}{4}\left[\frac{\rho}{4 N_{0}}\right]^{-\eta_{\min } \cdot M_{R}} \sum_{\mathbf{e} \in \mathcal{E}_{\min }}\left[\prod_{k=1}^{l} \prod_{i=1}^{\eta_{k}} \lambda_{i}^{(k)} u_{k}\right]^{-M_{R}}$,

where $\eta_{\text {min }}=\min _{\forall \mathbf{e}} \sum_{k=1}^{l} \eta_{k}$ and $\eta_{k}$ is the rank of the transformed STC channel matrix defined in [7, equation (10)] for the $k$-th transmission. The maximum diversity gain that can be achieved is $\max \left\{\eta_{\min }\right\}=L M_{T}$ when $\eta_{k}=M_{T}$. Substituting $\eta_{k}$ with $M_{T}$ leads to Proposition 2.

We note that the maximum diversity gains for the type I and the type II Hybrid-ARQ are same. The packet combining does not increase the diversity order.

From equations (10) and (11), the unified approximation of the PER for STC Hybrid-ARQ schemes with maximum diversity order at high SNR (Signal-to-Noise Ratio) can be written as

$$
P_{l}\left(E_{1}, \cdots, E_{l}\right) \approx A_{l} \prod_{k=1}^{l}\left(\frac{E_{k}}{N_{0}}\right)^{-D_{k}},
$$

where the bound for $A_{l}$ for the type I and the type II HybridARQ can be obtained from equations (10) and (11). In the remainder of this paper, we will use the above expression for the transmission probability in deriving optimal power allocation strategies for the Hybrid-ARQ system.

\section{OPTIMAL POWER ALLOCATION}

The optimal power allocation problem that minimizes the average power under a PER constraint can be formulated as

$\min _{E_{1}, E_{2}, \cdots, E_{L}}\left\{\bar{E}=\sum_{l=1}^{L} E_{l} P_{l-1}\left(E_{1}, \cdots, E_{l-1}\right)\right\}$

subject to $P_{L}\left(E_{1}, \cdots, E_{L}\right) \leq P_{e}^{\max }, E_{l}>0, l=1, \cdots, L$,

where $P_{0}=1$ by definition.

The above optimization problem can be written as a GP problem [8]-[10] in the standard primal form after applying approximation (12).

$$
\begin{aligned}
\min _{\underline{x}} & \left\{f_{0}(\underline{x})=x_{1}+A_{1} x_{2} x_{1}^{-D_{1}}+A_{2} x_{3} x_{1}^{-D_{1}} x_{2}^{-D_{2}}+\cdots\right. \\
& \left.+A_{L-1} x_{L} \prod_{l=1}^{L-1} x_{l}^{-D_{l}}\right\}
\end{aligned}
$$

subject to $A_{L} \prod_{i=1}^{L} x_{l}^{-D_{l}} \leq P_{e}^{\max }, x_{l}>0, l=1, \cdots, L$,

where $\underline{x}=\left(\begin{array}{llll}x_{1} & x_{2} & \cdots & x_{L}\end{array}\right), x_{l}=E_{l} / N_{0}, \forall l=1, \cdots, L$ and $D_{l}$ is the incremental diversity gain for the $l$-th ARQ round. This is a GP with a zero degree of difficulty and thus has a unique solution. Because of its special form, we can obtain the closed-form solution by solving its dual program, which is formulated as

$$
\max _{\underline{\delta}}\left\{v(\underline{\delta})=\prod_{l=1}^{L}\left(\frac{A_{l-1}}{\delta_{l}}\right)^{\delta_{l}}\left(\frac{A_{L}}{P_{e}^{\max }}\right)^{\delta_{L+1}}\right\}
$$

subject to $\sum_{l=1}^{L} \delta_{l}-1=0, \delta_{l}-\sum_{j=l+1}^{L+1} \delta_{j} D_{l}=0, l=1, \cdots, L$,

where $\underline{\delta}=\left(\delta_{1}, \cdots, \delta_{L+1}\right)$ is the design variable for the dual 
program, which can be solved from the linear constraints as

$$
\begin{aligned}
\delta_{l}^{*} & =\frac{D_{l} \prod_{j=l+1}^{L}\left(1+D_{j}\right)}{\prod_{j=1}^{L}\left(1+D_{j}\right)-1}, \quad l=1, \cdots, L-1, \\
\delta_{L}^{*} & =\frac{D_{L}}{\prod_{j=1}^{L}\left(1+D_{j}\right)-1}, \\
\delta_{L+1}^{*} & =\frac{1}{\prod_{j=1}^{L}\left(1+D_{j}\right)-1} .
\end{aligned}
$$

From the primal dual relation, the primal program and its optimal solution $\underline{x}^{*}=\left(x_{1}^{*}, x_{2}^{*}, \cdots, x_{L}^{*}\right)$ satisfy

$$
f_{0}\left(\underline{x}^{*}\right)=v\left(\underline{\delta}^{*}\right)=\prod_{l=1}^{L}\left(\frac{A_{l-1}}{\delta_{l}^{*}}\right)^{\delta_{l}^{*}}\left(\frac{A_{L}}{P_{e}^{\max }}\right)^{\delta_{L+1}^{*}},
$$

where $\underline{\delta}^{*}=\left(\delta_{1}^{*}, \delta_{2}^{*}, \cdots, \delta_{L+1}^{*}\right)$.

Let $u_{l}(\underline{x})=A_{l-1} x_{l} \prod_{j=1}^{l-1} x_{j}^{-D_{j}}$, then $f_{0}(\underline{x})=u_{1}(\underline{x})+$ $u_{2}(\underline{x})+\cdots+u_{L}(\underline{x})$, and $\delta_{l}=u_{l}(\underline{x}) / f_{0}(\underline{x})$ is defined as the proportion of the average energy consumption for the $l$-th ARQ round relative to the total energy consumption. According to the duality theory [8, Theorem 1 (iv)], we have

$$
u_{l}\left(\underline{x}^{*}\right)=v\left(\underline{\delta}^{*}\right) \delta_{l}^{*}=f_{0}\left(\underline{x}^{*}\right) \delta_{l}^{*}, \quad l=1,2, \cdots, L .
$$

And the optimal solution satisfies

$$
\begin{aligned}
x_{1}^{*} & =f_{0}\left(\underline{x}^{*}\right) \delta_{1}^{*}, \\
x_{l}^{*} & =\frac{A_{l-2} D_{l}}{A_{l-1} D_{l-1}\left(1+D_{l}\right)}\left(x_{l-1}^{*}\right)^{\left(1+D_{l-1}\right)}, l=2, \cdots, L .
\end{aligned}
$$

Note that the PER approximation does not hold for SNR $<1$ and, therefore, the optimization scheme cannot be applied with extremely low SNRs. Also note that the optimization scheme does not change the diversity gain, because the incremental diversity gain for each ARQ round is a constant. The theoretical bound for the $A_{l}$ is not tight enough to derive the accurate value of the $A_{l}$. Therefore, it is obtained from the PER statistics of the channel measurement or simulations in practice.

\section{A. Impact of the diversity gain}

To simplify the notation, we assume equal incremental diversity gains $D_{l}=D, l=1, \cdots, L$ and either the number of ARQ rounds $L$ or $D$ is large. For conventional HybridARQ with equal power allocation for each ARQ round, i.e., $x_{1}=x_{2}=\cdots=x_{L}=\rho$ and a target PER $P_{e}$, based on equation (2) and (12), the average power $E_{a v}^{N P C}$ is calculated as

$$
E_{a v}^{N P C}=\rho \sum_{l=1}^{L} A_{l-1} \rho^{-D(l-1)},
$$

where the transmit power $\rho=\left(\frac{P_{e}}{A_{L}}\right)^{-\frac{1}{D L}}$, which comes from the PER constraint $A_{L} \prod_{i=1}^{L} x_{l}^{-D} \leq P_{e}$ and $A_{0}=1$ by definition. We use superscript $N P C$ to represent no power control case.

For the optimal power allocation scheme and a large D, we can approximate the equation (16) as

$$
\delta_{l}^{*} \approx \frac{D}{(1+D)^{l}}, \quad l=1,2, \cdots, L .
$$

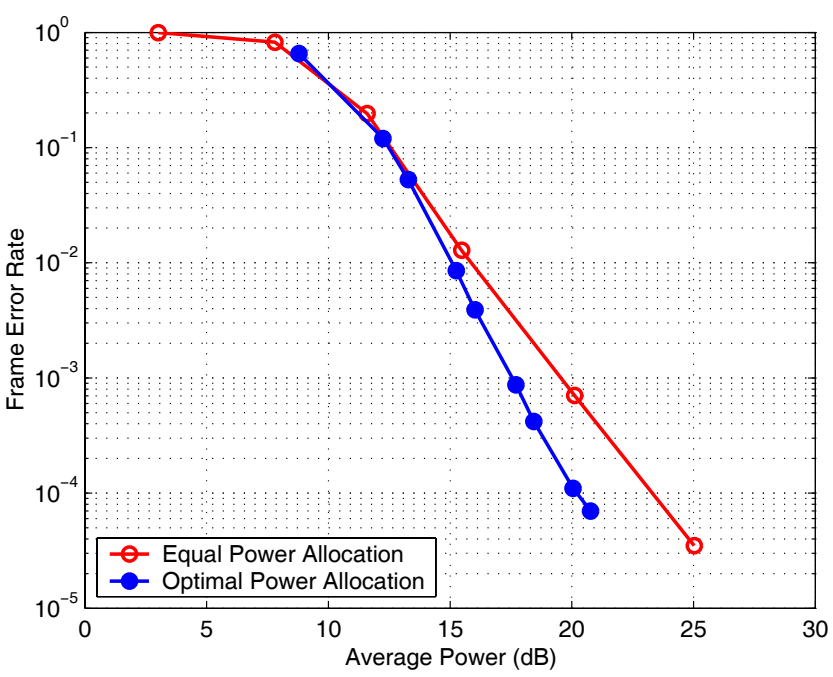

Fig. 2. PER for equal power allocation scheme and optimum power allocation scheme for type I Hybrid ARQ.

With the equal power allocation scheme and a large D,

$$
\delta_{l}^{N P C} \approx A_{l-1} \rho^{-D(l-1)}, \quad l=1,2, \cdots, L .
$$

For both optimal and equal power allocation schemes, with larger incremental diversity gain, i.e., larger number of transmitter and receiver antennas, more energy is allocated to the first ARQ round, i.e., $\delta_{1}^{*} \rightarrow 1$ and $\delta_{1}^{N P C} \rightarrow 1$ as $D \rightarrow \infty$. Hence the Hybrid-ARQ scheme converges to a single transmission scheme with an increase of the diversity gain. This fact suggests that from the energy saving point of view, a single transmission scheme is good enough for a system with large diversity gain. Compared with the $\delta_{l}^{*}, \delta_{l}^{N P C}$ converges faster as a function of $D$. Hence, as the incremental diversity gain increases, the conventional Hybrid-ARQ scheme converges faster to a single transmission scheme than the optimal scheme in terms of the average energy consumption, and both Hybrid-ARQ schemes do not provide much energy savings over single transmission schemes. Therefore, the performance gain of an optimal power allocation scheme over an equal power allocation scheme diminishes as incremental diversity gain increases.

\section{APPLICATIONS}

In the following, we apply the optimal power allocation scheme in simulated Hybrid-ARQ systems. To illustrate the performance gain against the no power control Hybrid-ARQ systems, we apply the type I Hybrid-ARQ and Chase combining Hybrid-ARQ over a 2x1 SIMO (Single Input Multiple Output) Rayleigh block fading channel with an 8-states 4-PSK STTC coding scheme defined in [11, Fig. 5]. The diversity and coding gain parameters of the PER, i.e., $A_{l}, D_{l}, l=1,2$ are obtained from the PER statistics based on simulations. We obtain PER curves for 5 cases, where the power difference between the two ARQ rounds is $-6 \mathrm{~dB},-3 \mathrm{~dB}, 0 \mathrm{~dB}, 3 \mathrm{~dB}$ and $6 \mathrm{~dB}$, and find the best fit for $A_{1}, D_{1}, A_{2}, D_{2}$. Next, we calculate the value of $x_{1}^{*}, x_{2}^{*}$ of a set of PER targets $P_{e}^{\max }=\{0.1,0.05,0.01,0.005,0.001,0.0005,0.0001\}$. For each set of $x_{1}^{*}, x_{2}^{*}$, we obtain the PER value by simulation 


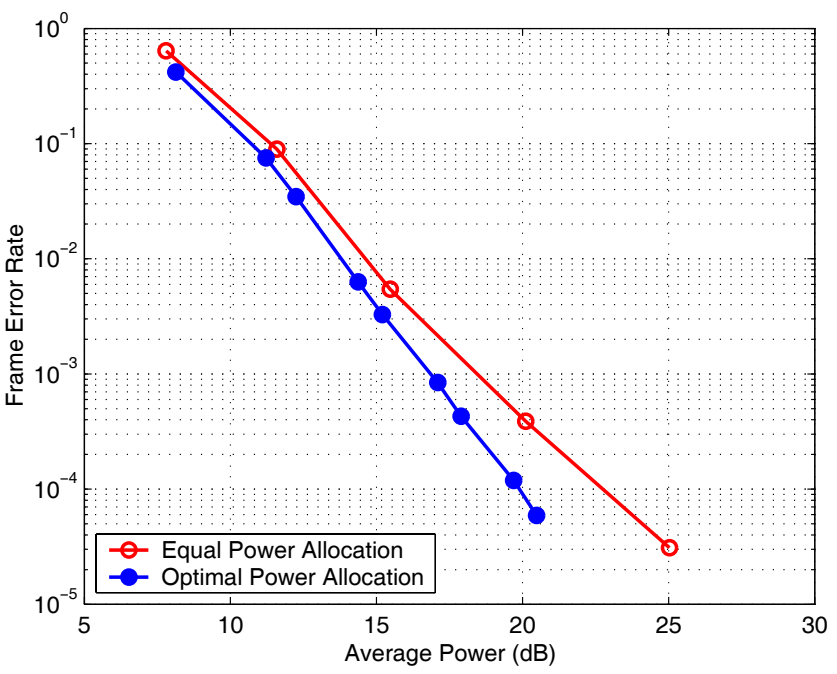

Fig. 3. PER for equal power allocation scheme and optimum power allocation scheme for Chase combining Hybrid ARQ.

to evaluate the power savings. The results are shown in Figure 2 and 3. Note that, for extremely high PER like 0.5, the optimization scheme is not applicable.

In Fig. 2 and Fig. 3, the results show that at a target PER of $10^{-4}$, the optimal power allocation scheme can provide a gain of up to $3 \mathrm{~dB}$ for the type I Hybrid-ARQ and $2.5 \mathrm{~dB}$ for the Chase combining Hybrid-ARQ.

\section{CONCLUSION}

In this paper, we have provided a method to minimize the average energy consumption for Hybrid-ARQ systems over an $L$-block Rayleigh fading channel while maintaining a target PER. The optimization problem was formulated and solved as a geometric programming problem. The Lagrangian dual formulation was used to derive closed-form expressions for the optimal objective function and the optimal power allocation.
Compared with a traditional Hybrid-ARQ scheme with equal power allocation for all ARQ rounds, the optimal scheme achieved significant power savings for channels with low diversity gains. The simulation results showed, at a target PER of $10^{-4}$, a gain of up to $3 \mathrm{~dB}$ for type I Hybrid-ARQ and $2.5 \mathrm{~dB}$ for Chase combining Hybrid-ARQ were achieved over an STTC (Space-Time Trellis Code) coded SIMO channel with a maximum of two (re)transmissions. The analytical results showed that a larger gain was achieved for a lower PER target, and the energy savings obtained from the optimization scheme decreased with a higher incremental diversity gain.

\section{REFERENCES}

[1] S.-H. Hwang, B. Kim, and Y.-S. Kim, "A Hybrid ARQ scheme with power ramping," in Proc. Veh. Technol. Conf. (VTC 2001), vol. 3, Oct. 2001, pp. 1579-1583.

[2] Z. Sun and X. Jia, "Energy efficient Hybrid ARQ scheme under error constraints," Wireless Personal Commun., vol. 25, pp. 307-320, 2003.

[3] H. E. Gamal, G. Caire, and M. O. Damen, "The MIMO ARQ channel: diversity-multiplexing-delay tradeoff," IEEE Trans. Inf. Theory, vol. 52, pp. 3601-3621, Aug. 2006.

[4] S. Lin, D. J. C. Jr., and M. J. Miller, "Automatic-repeat-request error control schemes," IEEE Commun. Mag., vol. 22, no. 12, pp. 5-17, Dec. 1984.

[5] D. M. Mandelbaum, "An adaptive-feedback coding scheme using incremental redundancy," IEEE Trans. Inf. Theory, vol. 20, pp. 388-389, May 1974.

[6] D. Chase, "Code combining-a maximum-likelihood decoding approach for combining an arbitrary number of noisy packets," IEEE Trans. Commun., vol. 33, pp. 385-393, May 1985.

[7] M. Chiani, A. Conti, and V. Tralli, "A pragmatic approach to spacetime coding," in Proc. IEEE International Conf. Commun. 2001 (ICC), vol. 9, June 2001, pp. 2794-2799.

[8] R. J. Duffin, E. L. Peterson, and C. Zener, Geometric Programming: Theory and Application. John Wiley \& Sons, Inc., 1967.

[9] S. Rao, Optimization Theory and Applications. John Wiley \& Sons, Inc., 1983.

[10] S. Boyd and L. Vandenberghe, Convex Optimization. Cambridge University Press, 2004.

[11] V. Tarokh, N. Seshadri, and A. R. Calderbank, "Space-time codes for high data rate wireless communication: performance criterion," IEEE Trans. Inf. Theory, vol. 44, pp. 744-765, Mar. 1998. 